\title{
Seizure Expression During Electroconvulsive Therapy: Relationships with Clinical Outcome and Cognitive Side Effects
}

\author{
Tarique D Perera*, ${ }^{1,2}$, Bruce Luber ${ }^{1,2}$, Mitchell S Nobler ${ }^{1,2}$, Joan Prudic ${ }^{1,2}$, Christopher Anderson ${ }^{1,2}$ \\ and Harold A Sackeim ${ }^{1,2,3}$
}

'Department of Biological Psychiatry, New York State Psychiatric Institute, New York, NY, USA; ${ }^{2}$ Department of Psychiatry, College of Physicians and Surgeons of Columbia University, New York, NY, USA; ${ }^{3}$ Department of Radiology, College of Physicians and Surgeons of Columbia University, New York, NY, USA

\begin{abstract}
Since electroconvulsive therapy (ECT) can result in generalized seizures that lack efficacy, physiological markers of treatment adequacy are needed. Specific electroencephalographic (EEG) features differentiate seizures produced with barely suprathreshold right unilateral (RUL) ECT, an ineffective treatment, from effective forms of ECT. This study determined whether EEG features are sensitive to treatment condition using a broad dosing range for RUL ECT, as well as predictive of clinical and cognitive outcomes. Quantitative EEG measures and observer ratings were compared in predictive power. From a larger study, 54 in-patients with major depression were randomized to low $(\mathrm{I} .5 \times$ seizure threshold $(\mathrm{ST}))$, moderate $(2.5 \times \mathrm{ST})$, or high-dose $(6 \times \mathrm{ST}) \mathrm{RUL} \mathrm{ECT}$, or high-dose $(2.5 \times \mathrm{ST})$ bilateral (BL) ECT. High dosage RUL and BL ECT were comparable in efficacy, and superior to low and moderate dosage RUL ECT. In the slow frequency bands $(\delta)$, BL ECT resulted in greater ictal power, ictal coherence, and postictal suppression than each RUL ECT condition, but the EEG measures failed to discriminate the RUL ECT groups. EEG measures were modestly associated with clinical outcome, with greater ictal power, $\delta$ coherence, and postictal suppression positive predictors. None of the EEG measures were associated with cognitive outcomes. Inability to distinguish forms of RUL ECT differing markedly in dosage and efficacy suggests that EEG measures have limited potential as markers of treatment adequacy. Rather than assaying treatment adequacy, the EEG features associated with efficacy may reflect individual differences in the strength of inhibitory processes that terminate the seizure, and can help isolate the biological variability that predisposes to positive or negative clinical response to ECT.

Neuropsychopharmacology (2004) 29, 813-825, advance online publication, 2 I Janauary 2004; doi: I 0.1038/sj.npp. I 300377
\end{abstract}

Keywords: electroconvulsive therapy; electroencephalogram; major depressive disorder; efficacy; amnesia; cognition; seizure

\section{INTRODUCTION}

Electroconvulsive therapy (ECT) is the most effective acute treatment for major depression (American Psychiatric Association, 2001). It was thought for decades that the generalized seizure provided the necessary and sufficient conditions for ECT's antidepressant effects (Ottosson, 1960; Fink, 1979). However, it is now established that generalized seizures lacking efficacy can be reliably produced (Robin and De Tissera, 1982; Sackeim et al, 1987a, 1993, 2000b; McCall et al, 2000), as clinical outcome is strongly influenced by the anatomic positioning of electrodes and

\footnotetext{
*Correspondence: Dr TD Perera, Department of Biological Psychiatry, New York State Psychiatric Institute, Unit 126, 105 I Riverside Drive, New York, NY 10032, USA. Tel: + I 212543 5274, Fax: + I 212543 5855, E-mail: tp I19@columbia.edu

Received 21 May 2003; revised 15 September 2003; accepted 12 November 2003

Online Publication: 2 December 2003 at http://www.acnp.org/citations/ Npp I2020303232/default.pdf
}

electrical dosage relative to seizure threshold (ST). Therefore, efficacy is dependent on intracerebral current paths and the current density within those paths, implicating anatomic specificity in the circuitry subserving ECT's antidepressant effects. Supporting this perspective, imaging studies have shown that distinct topographic changes in brain activity are associated with ECT's therapeutic and adverse cognitive effects (Nobler et al, 1994, 2000, 2001; Sackeim et al, 1996, 2000a).

Given that the patients who receive ECT are often the most severely ill or those with established treatment resistance, ensuring adequate delivery is especially critical. Yet, previous guidelines, requiring essentially a generalized seizure of sufficient duration, proved to have limited relevance for efficacy. Consequently, there is great interest in identifying physiological markers that predict clinical outcomes with sufficient accuracy to guide alterations in treatment parameters (eg stimulus dosing, switching electrode placements, etc) (Krystal and Weiner, 1994). 
Several potential indices have been examined. ECT produces an acute surge in plasma catecholamines, cortisol, growth hormone, luteinizing hormone, neurophysin, oxytocin, prolactin, vasopressin, and other hormones and peptides (Whalley et al, 1987; Devanand et al, 1989; Scott, 1989; Kronfol et al, 1991; Devanand et al, 1998; Fink and Nemeroff, 1989), and an acute decrease in plasma gamma aminobutyric acid (GABA) (Devanand et al, 1995). However, none of the acute biochemical changes have consistent associations with efficacy (Sackeim et al, 1995). The most studied biochemical measure, the acute prolactin surge (Ohman et al, 1976; Zis et al, 1996; Lisanby et al, 1998; Sundblom et al, 1999), is reduced when right unilateral (RUL) ECT is administered with dosage barely above ST, an ineffective form of treatment (Zis et al, 1996; Lisanby et al, 1998). Nonetheless, the magnitude of this surge is generally found to be unrelated to clinical outcome (Lisanby et al, 1998).

Electroencephalographic (EEG) activity during and following the seizure has received the most attention. The EEG has the following phases: recruitment (initial low amplitude, fast frequency activity), tonic (high amplitude, fast frequency, spike, and polyspike activity), clonic (high amplitude spike and slow wave complexes), termination (progressive change in slow wave amplitude and/or frequency), and immediate postictal silence (bioelectric suppression following seizure termination) (Staton et al, 1981; Weiner, 1982). Patients vary in the duration, strength, and morphology of these peri-ictal phases. For example, with increasing age, the intensity of seizure expression decreases, particularly the amplitude of slow wave $(\delta)$ activity, and there is less postictal suppression (Nobler et al, 1993, 2000; Krystal et al, 1995, 2000b; Luber et al, 2000).

Ictal and immediate postictal EEG parameters distinguish RUL ECT administered either just above $(1 \times \mathrm{ST})$ or moderately above threshold (2.25-2.5 $\times$ ST). Higher dosage treatment produces more intense ictal EEG expression and greater postictal suppression (Krystal et al, 1993, 1995, 2000b; Nobler et al, 1993, 2000; Luber et al, 2000). Consistent with this pattern, several studies found that patients with superior clinical outcome had greater ictal amplitude, especially in the $\delta$ frequency band, and greater postictal suppression (Nobler et al, 1993, 2000; Krystal et al, 1995, 2000a, b; Folkerts, 1996; Suppes et al, 1996; Luber et al, 2000). ECT devices now provide quantitative analyses of the ictal and postictal EEG, in the form of power and coherence values for specific frequency bands, or as composite indices intended to quantify 'seizure adequacy' and predict treatment outcome. The common recommendation is that an increase in stimulus dosage be considered when ictal expression is weak and/or when postictal suppression is absent, and especially when coupled with slow or incomplete clinical progress (American Psychiatric Association, 2001).

This translation of the early findings into practice recommendations may be premature. The evidence that EEG features distinguish ineffective and more effective forms of ECT or predict clinical outcome mainly derives from studies contrasting barely suprathreshold and moderately suprathreshold RUL ECT (Krystal et al, 1993, 1995; Nobler et al, 1993, 2000). With respect to efficacy, the doseresponse function for RUL ECT is wide, and efficacy appears to be maximized, matching that of bilateral (BL) ECT, only at high dosage relative to ST (eg $6 \times \mathrm{ST})(\mathrm{McCall}$ et al, 2000; Sackeim et al, 2000b; Heikman et al, 2002; Tew et al, 2002). It is not known whether peri-ictal EEG indices show progressive change across the dosage range that impacts on efficacy, or whether the EEG manifestations saturate at dosage levels that result in inferior efficacy. Indeed, one small within-patient study found that observer ratings of seizure quality distinguished barely suprathreshold and moderately above threshold RUL ECT, but did not separate moderate and high dosage RUL ECT (McCall et al, 1998). It has also been assumed that, given significant predictive associations between EEG indices and clinical outcome, stimulus dosing should be guided by EEG findings of seizure 'adequacy'. Yet, no prospective study has shown that these associations have sufficient sensitivity and specificity to aid clinical decision-making. The relations of the peri-ictal EEG measures to the major side effects of ECT are also not known. No study has examined the associations with cognitive side effects. Depending on the relative strength of associations, the cognitive 'costs' of changing treatment parameters to produce adequate EEG seizure expression may or may not outweigh the 'benefits' of somewhat higher remission rates.

We reported a randomized, double-masked trial contrasting three dosages of RUL ECT $(1.5,2.5$, or $6 \times \mathrm{ST})$ and a high dosage form of BL ECT $(2.5 \times \mathrm{ST})$ (Sackeim et al, $2000 \mathrm{~b})$. High dosage RUL $(6 \times \mathrm{ST})$ and BL ECT did not differ in any efficacy measure, while the two lower dosages of RUL ECT had inferior outcome. A cognitive battery was administered at each treatment, as well as before, during, and after the ECT course. We report the first examination of whether quantitative EEG measures are sensitive to a wide range of dosage in patients administered RUL ECT, whether these indices are associated with clinical outcome independent of the form of ECT, and the extent to which ictal and postictal EEG features covary with cognitive effects.

Fast Fourier transformation (FFT) and other algorithms can produce quantitative measures of seizure expression not readily assessed by visual inspection. On the other hand, trained raters may make reliable determinations about periictal features that are difficult to capture in an automated algorithm. This may especially hold when pattern matching is key, such as evaluating seizure stereotypy or abruptness of termination (McCall et al, 1995). In some cases, trained raters may be less influenced by common artifacts, such as slow-wave motion artifact, due to ventilation of the patient, obscuring postictal suppression. Thus, another goal was to contrast quantitative and observer ratings of peri-ictal EEG features in their associations with the clinical and cognitive outcomes of ECT.

\section{METHODS}

\section{Patients}

Study procedures have been described elsewhere (Sackeim et al, 2000b). Patients met the Research Diagnostic Criteria (Spitzer et al, 1978) for major depressive disorder and DSMIV criteria for major depressive episode based on interviews using the Schedule for Affective Disorders and Schizophrenia (Endicott and Spitzer, 1978). Inclusion criteria also 
included a score of at least 18 on the Hamilton Rating Scale for Depression (HRSD, 24-item) (Hamilton, 1967) and willingness to provide informed consent. Patients were excluded with a history of schizophrenia, schizoaffective disorder, other nonmood disorder psychosis, rapid-cycling bipolar disorder, neurological illness or insult, alcohol or other drug abuse within the past year, ECT within the past 6 months, or severe medical illness. The Institutional Review Boards of both the New York State Psychiatric Institute and Columbia University approved the study.

Digital recording of the EEG at each treatment was started after the first 26 of 80 patients had completed the parent study. Therefore, the sample for this substudy comprised 54 patients. With the exception of lorazepam (up to $3 \mathrm{mg} / \mathrm{day}$ PRN), patients were withdrawn from psychotropic medication at least 5 days before ECT. Imposing an upper limit of 30 days, the average washout before ECT (mean \pm SD) was $15.1 \pm 8.1$ days, and did not differ among the four treatment groups, $\mathrm{F}(3,50)=0.46, P=0.71$ (Table 1). During the ECT course, the average daily dose of lorazepam was $1.2 \pm 1.1$, with no difference among the treatment groups, $\mathrm{F}(3,50)=0.23, P=0.88$.

\section{Electroconvulsive Therapy}

Patients and staff not involved in ECT administration were masked to treatment condition. Patients were randomized to the four treatment conditions, stratified by whether or not they had received an adequate antidepressant medication trial during the index episode, using Antidepressant Treatment History Form (ATHF) criteria (Sackeim, 2001). Atropine $(0.4 \mathrm{mg})$, methohexital $(0.75 \mathrm{mg} / \mathrm{kg})$, and succinylcholine $(0.75 \mathrm{mg} / \mathrm{kg})$ were the anesthetic medications. The bifrontotemporal and d'Elia placements were used for BL and RUL ECT, respectively (American Psychiatric Association, 2001). ECT was administered three times per week with a customized MECTA SR1 device (MECTA Corp.,
Tualatin, Oregon). ST was quantified at the first and last treatments using the empirical titration procedure (Sackeim et al, 1987b). At all other treatments, stimulus intensity was 50,150 , or $500 \%$ above initial ST for the low, moderate, and high dosage RUL groups, respectively, and 150\% above threshold in the high dosage BL group. Frequency and train duration were the electrical parameters manipulated, with pulse width $(1.5 \mathrm{~ms})$ and amplitude $(0.8 \mathrm{~A})$ constant. As a primary goal of the parent study was determine whether a high dosage form of RUL ECT could match the efficacy of BL ECT, it was important to use as a 'gold standard' a robust form of BL ECT. BL ECT at $150 \%$ above ST $(2.5 \times \mathrm{ST})$ is at the upper limit of dosage for routine use and its cognitive side effects are likely to be especially pronounced.

\section{Quantitative EEG Measures and Observer Ratings}

EEG was recorded with left and right frontal (approximating Fp1 and Fp2) leads. The lower boundary of the frontal sites was $2.54 \mathrm{~cm}$ above the midpoint of each eyebrow. The reference electrodes were positioned high on the ipsilateral mastoid process to avoid pulse artifact. A common ground electrode was placed in the middle of the forehead, midway between the frontal electrodes. The EEG sites were cleansed with alcohol, dried, and then abraded to reduce impedance (Redux Paste, Hewlett Packard Co., Andover, MA). Prelubricated, self-adhesive, disposable pediatric ECG electrodes (ConMed Corp., Utica, NY) were used for the EEG electrodes. Impedance at each of the five sites was recorded at each treatment, and electrodes were reapplied when impedance exceeded $10 \mathrm{k} \Omega$.

The MECTA SR1 amplified the two EEG leads and provided hard copy tracings. The amplified analog EEG signals were fed from the SR1 to a 12-bit analog/digital converter and digitized at a rate of 500 samples/s. A masked technician inspected the EEG data off-line and removed

Table I Demographic and Clinical Characteristics of the Sample

\begin{tabular}{|c|c|c|c|c|}
\hline & $\begin{array}{l}\text { Low-dose RUL } \\
\text { ECT }(N=13)\end{array}$ & $\begin{array}{l}\text { Medium-dose RUL } \\
\qquad E C T(N=17)\end{array}$ & $\begin{array}{l}\text { High-dose RUL } \\
\text { ECT }(N=I I)\end{array}$ & $\begin{array}{l}\text { High-dose BL } \\
\text { ECT }(N=13)\end{array}$ \\
\hline Age (years) & $61.8 \pm 17.3$ & $57.3 \pm 18.0$ & $47.3 \pm 17.3$ & $50.5 \pm 12.1$ \\
\hline Gender (N, \% female) & $8(6 \mid .5)$ & I | (64.7) & $8(72.7)$ & $7(53.8)$ \\
\hline Unipolar (N, \%) & $10(76.9)$ & $10(58.9)$ & $7(63.6)$ & $10(76.9)$ \\
\hline Psychotic depression (N, \%) & $5(38.5)$ & $5(29.4)$ & $4(36.4)$ & $2(15.4)$ \\
\hline Medication resistant (N, \%) & $8(6 \mid .5)$ & I | (64.7) & $7(63.6)$ & $9(69.2)$ \\
\hline Duration current episode (weeks) & $52.1 \pm 57.1$ & $72.5 \pm 83.0$ & $66.8 \pm 75.3$ & $70.1 \pm 119.0$ \\
\hline Number of previous episodes ${ }^{\mathrm{a}}$ & $2.4 \pm 2.1$ & $3.1 \pm 2.1$ & $3.2 \pm 2.8$ & $3.1 \pm 3.0$ \\
\hline $\begin{array}{l}\text { Number of previous psychiatric } \\
\text { hospitalizations }^{\text {a }}\end{array}$ & $2.1 \pm 2.7$ & $2.5 \pm 2.0$ & $1.4 \pm 3.1$ & $1.3 \pm 1.7$ \\
\hline Age at onset (years) & $43.2 \pm 16.5$ & $36.5 \pm 20.4$ & $29.8 \pm 12.1$ & $34.1 \pm 17.9$ \\
\hline Previous history of ECT (N, \%) & $4(30.8)$ & $7(4 \mid .2)$ & $2(18.2)$ & $3(23.1)$ \\
\hline Length of pre-ECT medication washout (days) & $16.0 \pm 8.6$ & $14.6 \pm 7.8$ & $13.1 \pm 7.6$ & $16.7 \pm 8.7$ \\
\hline Baseline HRSD & $31.7 \pm 7.1$ & $28.8 \pm 5.7$ & $31.7 \pm 6.4$ & $28.2 \pm 7.5$ \\
\hline Baseline GAF & $35.8 \pm 11.6$ & $41.9 \pm 9.1$ & $42.9 \pm 10.3$ & $39.2 \pm 11.4$ \\
\hline
\end{tabular}

HRSD = Hamilton Rating Scale for Depression; GAF = Global Assessment of Function.

a Number of previous episodes and psychiatric hospitalizations capped at 10. 
epochs (each $1 \mathrm{~s}$ ) containing artifact. Starting at the point of seizure termination determined by the technician, the first $15 \mathrm{~s}$ was taken as the immediate postictal period. An FFT, with a resolution of $1.0 \mathrm{~Hz}$, was conducted on the epochs. Consecutive epochs overlapped by $0.5 \mathrm{~s}$ and were tapered with a Hanning function. For each channel, absolute power $\left(\mu \mathrm{V}^{2}\right)$ and coherence per epoch were computed within four frequency bands: $\delta(1.5-3.5 \mathrm{~Hz}), \theta(3.5-7.5 \mathrm{~Hz}), \alpha(7.5-$ $12.5 \mathrm{~Hz})$, and $\beta(12.5-28.5 \mathrm{~Hz})$. Power and coherence values were then averaged separately for the left and right channels over the epochs starting from the offset of the electrical stimulus until seizure termination (ictal values), and for the postictal period. Total power across frequency bands (global power) was calculated by summing the values of each band. The quantitative EEG indices were derived by averaging each measure across all treatments the patient received, excluding the first and last treatments which involved dosage titration. Power values were subjected to logarithmic transformation prior to statistical analysis.

One set of ancillary analyses retested the relationships with ictal power values, examining only the seven consecutive epochs (ie $4 \mathrm{~s}$ ) with greatest ictal power. These values reflected the peak ictal expression, with the maxima determined at each treatment separately for each frequency band and for left and right channels. These values were also averaged across all treatments, excluding titration sessions. Another set of ancillary analyses restricted the EEG data to the second and third treatments.

A masked rater applied the scales developed by Nobler et al (1993) to each EEG tracing $(N=449)$. Seizure duration was evaluated, as well as the strength (amplitude) of ictal expression and the stereotypy of the ictal pattern. The ictal termination phase was rated for the extent of amplitude and frequency reduction in slow wave activity, as well as the abruptness of the seizure ending. Finally, the extent of postictal suppression was rated. All ratings were based on the right channel. The right channel was selected since RUL ECT often shows asymmetry in EEG ictal and postictal expression, with greater EEG changes over the stimulated (right) than nonstimulated hemisphere. Like the quantitative measures, the ratings were averaged across the treatment course, excluding titration sessions.

\section{Clinical Outcome}

A masked clinical evaluation team completed the HRSD at least twice weekly and determined the number of treatments administered. ECT was continued until patients were asymptomatic or had not shown improvement over at least two consecutive treatments. To be classified as a nonresponder, patients had to receive at least 10 treatments. This criterion was reduced to eight treatments for patients who showed minimal improvement (HRSD percentage change from baseline $<20 \%$ ). The criteria for initial clinical response were a reduction in HRSD score from baseline of at least $60 \%$ and a maximal immediate post-ECT score of 16. Initial remitters had a post-ECT HRSD score of 10 or less. Final responders and remitters continued to meet the response and remission criteria 1 week following ECT, while free of psychotropics. Inter-rater reliability of HRSD ratings exceeded 0.95 at all time-points (intraclass correlation coefficients).

\section{Cognitive Evaluations}

Cognitive measures were selected a priori from a larger neuropsychological battery (Sackeim et al, 1986, 2000b, 1993). These tests assessed acute disorientation and retrograde amnesia (four acute measures), and short-term effects on global cognitive status, verbal and nonverbal anterograde amnesia, and retrograde amnesia for autobiographical information (five short-term measures).

Patients memorized a set of six words, two geometric shapes and two nonsense shapes a few minutes before each treatment. Learning was tested after presentation of each stimulus set (Sackeim, 1986). Following seizure termination and eyes opening upon command, recovery of orientation was assessed continuously for $90 \mathrm{~min}$ (Sobin et al, 1995). Patients who did not meet criteria for full recovery at this time limit were given scores of $100 \mathrm{~min}$. At $5 \mathrm{~min}$ after orientation criteria were met, a neuropsychological battery was administered, including assessment of memory for the previously learned material (Sackeim, 1986; Prudic et al, 1994). For each task, 12 equivalent stimuli sets were available. Retrograde amnesia was scored for each of the three stimulus categories as the percentage of items not recalled or recognized at postictal testing relative to the number of items recalled or recognized when testing immediate learning prior to the treatment. The acute disorientation score and each of the three amnesia scores were averaged across the same treatments as the EEG measures.

A neurocognitive battery was administered before ECT, the day after the sixth or seventh treatment, 2-7 days following the ECT course, and at long-term follow-up. This study focused on changes in cognitive function during the week following ECT relative to pre-ECT baseline. Associations with EEG indices at long-term follow-up are unlikely unless there are associations during the week following ECT, when deficits are greater.

The modified Mini-Mental State Examination (mMMS) (Stern et al, 1987), an expanded version (range 0-57) of the original MMS, assessed global cognitive status. Percentage change from baseline was the dependent measure. Nonverbal anterograde amnesia was assessed with complex figure reproduction (Rey, 1941; Spreen and Strauss, 1998). The percentage change in $20 \mathrm{~min}$ delayed reproduction scores from pre-ECT baseline was the dependent measure. The Buschke Selective Reminding Test (SRT) used alternative 12 -word lists and 10 trials to examine anterograde amnesia for verbal material (Buschke, 1973). After standard administration, free recall was assessed after a 2-h delay, and then the full SRT procedure was repeated. The dependent measure was the percentage change from preECT baseline in the total recall score over the delayed 10trial procedure. Retrograde amnesia for autobiographical information was tested using the Columbia University Autobiographical Memory Interview (AMI) (Sackeim et al, 1993; McElhiney et al, 1995; Sobin et al, 1995). With probes for approximately 200 details about personal experiences, the AMI retrograde amnesia scores were the percent of items reported at retesting that were inconsistent in factual detail with pre-ECT reports, and the percent of 'don't remember' responses for the factual items recalled at baseline. 


\section{Statistical Methods}

The patients assigned to the four treatment conditions were compared in baseline clinical and demographic characteristics using one-way analyses of variance (ANOVAs) for continuous measures and $\chi^{2}$ analyses for dichotomous variables. The treatment conditions were also compared in ECT treatment parameters and seizure duration measures using analyses of covariance (ANCOVAs) on continuous measures, with the four treatment modalities as a betweensubject factor and age as a covariate. For post hoc analyses, significant main effects of treatment modality were followed by $t$-tests on least-squares adjusted means. Age was used as a covariate since it has a strong effect on EEG seizure manifestations, seizure duration, and ST (Sackeim et al, 1987b; Nobler et al, 1993; Boylan et al, 2000). The four treatment conditions were compared in efficacy with an ANOVA on the percentage change in HRSD scores over the treatment course, using treatment modality as a betweensubject factor, and $\chi^{2}$ analyses contrasting the treatment groups in rates of initial and final response and remission.

Pearson product-moment correlations were used to examine the relations between the quantitative peri-ictal EEG indices and the observer ratings. The extent to which the four forms of ECT differed in the EEG indices was tested with ANCOVAs on each EEG measure, using treatment condition as a between-subject factor and age as a covariate. To determine whether specific quantitative EEG indices or observer ratings were related to clinical outcome independent of the effects of treatment modality, the percentage change in HRSD scores from baseline to immediately following the ECT course was added as a covariate to the earlier ANCOVAs. To determine the durability of relations with clinical outcome, the ANCOVAs were repeated substituting as a covariate the percentage change in HRSD scores from baseline to 1 week following ECT.

A similar strategy was used to examine the relations of the quantitative EEG indices and observer ratings to the acute and short-term cognitive effects of ECT. ANCOVAs, with treatment condition as the between-subject factor and age and EEG measure (quantitative or observer rating) as covariates, were conducted on each of the neurocognitive outcome measures.

Using the quantitative measures and observer ratings most strongly related to clinical improvement, discriminant functions were developed predicting initial or final remission status (Huberty, 1984). A backward elimination procedure restricted the final discriminant functions to those variables contributing significantly to outcome prediction.

The analyses examining relations with efficacy and cognitive effects were repeated, changing the quantitative EEG measures or restricting the data to two treatments early in the course. In one set of ancillary analyses substituted the power values for peak ictal expression to determine whether averaging across the total seizure obscured relations with outcomes. The second set of ancillary analyses repeated the primary analyses restricting the EEG data to the second and third treatments. By using repeated observation and averaging over the treatment course, the reliability of measurement was increased in the primary analyses, and thus the potential for significant associations. Furthermore, clinical utility of peri-ictal EEG measures is contingent on sensitivity to treatment conditions and/or accuracy in prediction of outcomes based on seizure manifestations at early treatments.

\section{RESULTS}

\section{Characteristics of the Sample}

There were no significant differences among the four treatment groups in the demographic and clinical characteristics presented in Table 1. The treatment groups also did not differ in anesthetic variables, motor or EEG measures of seizure duration, or the number of treatments administered (Table 2). As expected, the treatment conditions differed in initial ST, $\mathrm{F}(3,49)=4.78, P=0.005$, which was higher with BL ECT compared to each RUL ECT condition (all $P$ 's $\leqslant 0.004$ ). Due to the study design, the treatment groups differed in the stimulus charge in nontitration sessions, $F(3,49)=18.88, P<0.0001$, and in the ratio of charge to initial ST, $\mathrm{F}(3,49)=632.12, P<0.0001$. For the absolute charge in nontitration sessions, all groups differed from each other $(P$ 's $\leqslant 0.003)$, except for the high dosage RUL and BL ECT groups $(P=0.18)$.

Table 2 presents the response and remission rates for the treatment conditions. As in the parent study, high dosage RUL and BL ECT were more effective than low or moderate dosage RUL ECT. The ANCOVA on percentage change in HRSD scores yielded a significant effect of modality, $\mathrm{F}(3,49)=3.05, P=0.04$. High dosage RUL and BL ECT had equivalent degrees of symptomatic change and both differed significantly from the lower dosage RUL ECT condition (all $P$ 's $<0.02$ ). The high dosage RUL and BL ECT conditions had higher rates of initial response $\left(\chi^{2}(1)=7.38\right.$, $P=0.007)$ and initial remission $\left(\chi^{2}(1)=8.73, P=0.003\right)$ than the low and moderate dosage RUL ECT conditions. These groups also differed in rates of final response $\left(\chi^{2}(1)=4.62, \quad P=0.03\right)$ and remission $\left(\chi^{2}(1)=4.42\right.$, $P=0.04$ ). Approximately $80 \%$ of patients were initial responders and remitters with high-dose RUL and BL ECT, in line with expectations when optimal forms of ECT are used (American Psychiatric Association, 2001). That approximately $15 \%$ of patients no longer met remission criteria 1 week following ECT, while medication free, was in line with the previous studies that reassessed clinical outcome at this interval (Sackeim et al, 1987a, 1993).

\section{Relations Between Visual Ratings and Quantitative EEG Measures}

Seizure duration was assessed both by the trained rater from hard copy of tracings and the technician who processed the digitized EEG. The correlation between these assessments was robust, $r=0.95, \mathrm{df}=52, P<0.0001$.

Table 3 presents the correlations between the observer ratings, conducted on the right EEG channel and, where appropriate, quantitative EEG measures for that channel. The observer rating of seizure duration was unrelated to all quantitative EEG measures. In contrast, the observer rating of ictal strength showed robust relations in all frequency bands with ictal spectral power, as well as positive associations with each measure of coherence. In contrast, 
Table 2 Treatment Parameters and Clinical Outcome Measures

\begin{tabular}{|c|c|c|c|c|}
\hline & $\begin{array}{l}\text { Low-dose RUL } \\
\text { ECT }(N=13)\end{array}$ & $\begin{array}{l}\text { Medium-dose RUL } \\
\text { ECT }(N=17)\end{array}$ & $\begin{array}{l}\text { High-dose RUL } \\
\text { ECT }(N=I I)\end{array}$ & $\begin{array}{l}\text { High-dose BL } \\
\text { ECT }(N=13)\end{array}$ \\
\hline Atropine (mg) & $0.42 \pm 0.02$ & $0.4 I \pm 0.02$ & $0.44 \pm 0.02$ & $0.41 \pm 0.02$ \\
\hline Succinylcholine (mg) & $58.0 \pm 5.1$ & $60.1 \pm 4.4$ & $57.8 \pm 5.6$ & $67.4 \pm 5.0$ \\
\hline Initial seizure threshold (mC) & $81.0 \pm 12.8$ & $74.2 \pm 11.0$ & $77.5 \pm 13.9$ & $116.4 \pm 12.6$ \\
\hline Motor seizure duration (s) & $46.2 \pm 2.3$ & $42.6 \pm 2.0$ & $45.2 \pm 2.5$ & $39.9 \pm 2.3$ \\
\hline EEG seizure duration (s) & $60.6 \pm 12.9$ & $52.7 \pm 11.8$ & $63.3 \pm 16.2$ & $53.4 \pm 15.2$ \\
\hline Number of treatments in course & $9.8 \pm 0.86$ & $9.3 \pm 0.73$ & $8.1 \pm 0.93$ & $8.2 \pm 0.84$ \\
\hline Percentage reduction in immediate post-ECT HRSD scores & $54.9 \pm 29.6$ & $41.1 \pm 37.5$ & $71.8 \pm 27.9$ & $71.2 \pm 32.1$ \\
\hline Initial responder $(N, \%)$ & $5(38.5)$ & $8(47.1)$ & $9(81.8)$ & $10(76.9)$ \\
\hline
\end{tabular}

HRSD = Hamilton Rating Scale for Depression.

Table 3 Correlations Between Observer Ratings and Quantitative EEG Analyses

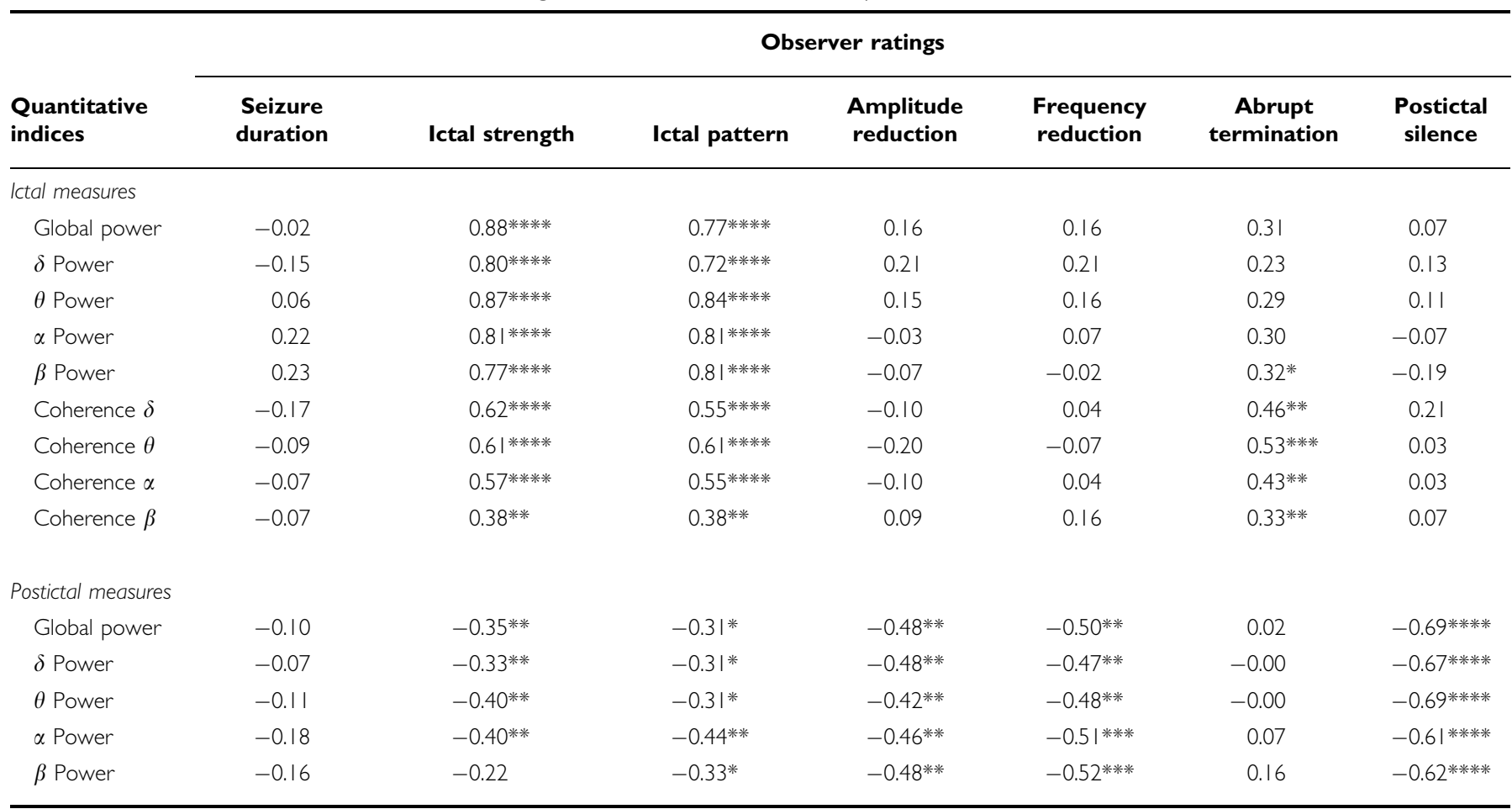

Only the right channel is included for power spectral values.

$* P<0.05$; ** $P<0.01$; *** $P<0.001$; ***** $P<0.000$ I.

the ictal strength ratings were inversely associated with all measures of postictal spectral power, indicating that ratings of greater ictal strength were associated with greater postictal suppression in the quantitative indices. This pattern was largely duplicated for the rating of ictal strength, although associations with postictal spectral power were somewhat weaker.

The observer rating of amplitude reduction during seizure termination was not associated with the quantitative ictal power and coherence measures, but had strong relations with all quantitative measures of postictal suppression. An identical pattern was observed for the rating of frequency reduction. The rating of the abruptness of seizure offset was not associated with spectral power either during or after the seizures, except for a modest association with ictal $\beta$ power. In contrast, abruptness of termination showed consistent associations with all quantitative measures of coherence. The observer rating of 
postictal silence showed consistent and robust inverse associations with all measures of postictal spectral power, and no associations with quantitative ictal measures. These findings indicate that a trained observer evaluating the ictal and postictal EEG can derive much of the same information as offered by quantitative analyses, and further that specific features detected by an observer covary with specific quantitative EEG indices.

\section{Relations Between ECT Modality and Quantitative EEG Measures}

Table 4 presents the findings from the ANCOVAs contrasting the four ECT modalities in the quantitative EEG measures. Ictal power differed among the modalities for global, $\delta$, and $\theta$ power in both the left and right channels. BL ECT resulted in greater power than each of the RUL conditions in all six comparisons. The modalities did not differ in ictal power in the higher frequency bands ( $\alpha$ or $\beta$ ) in either the left or right channels.

Among the coherence measures, main effects of modality were obtained only for ictal $\delta$. BL ECT had the highest $\delta$ coherence, differing from low dosage RUL ECT. For the postictal quantitative measures, significant effects of modality were only observed for postictal $\delta$ power (left and right) and $\theta$ power (right). In each case, BL ECT resulted in the greatest degree of postictal suppression, and differed significantly from low dosage RUL ECT, which had the least degree of suppression. In addition, in each case, low dosage RUL ECT differed from moderate dosage RUL ECT in manifesting less postictal suppression.

Thus BL ECT differed from the RUL ECT conditions by displaying greater ictal power in the slower frequency bands, greater $\delta$ coherence, and greater postictal suppression in the slow frequency bands. In contrast, there were few differences among the RUL ECT conditions and no indication that high dosage RUL ECT was distinct. In

Table 4 Quantitative EEG Measures as a Function of ECT Modality

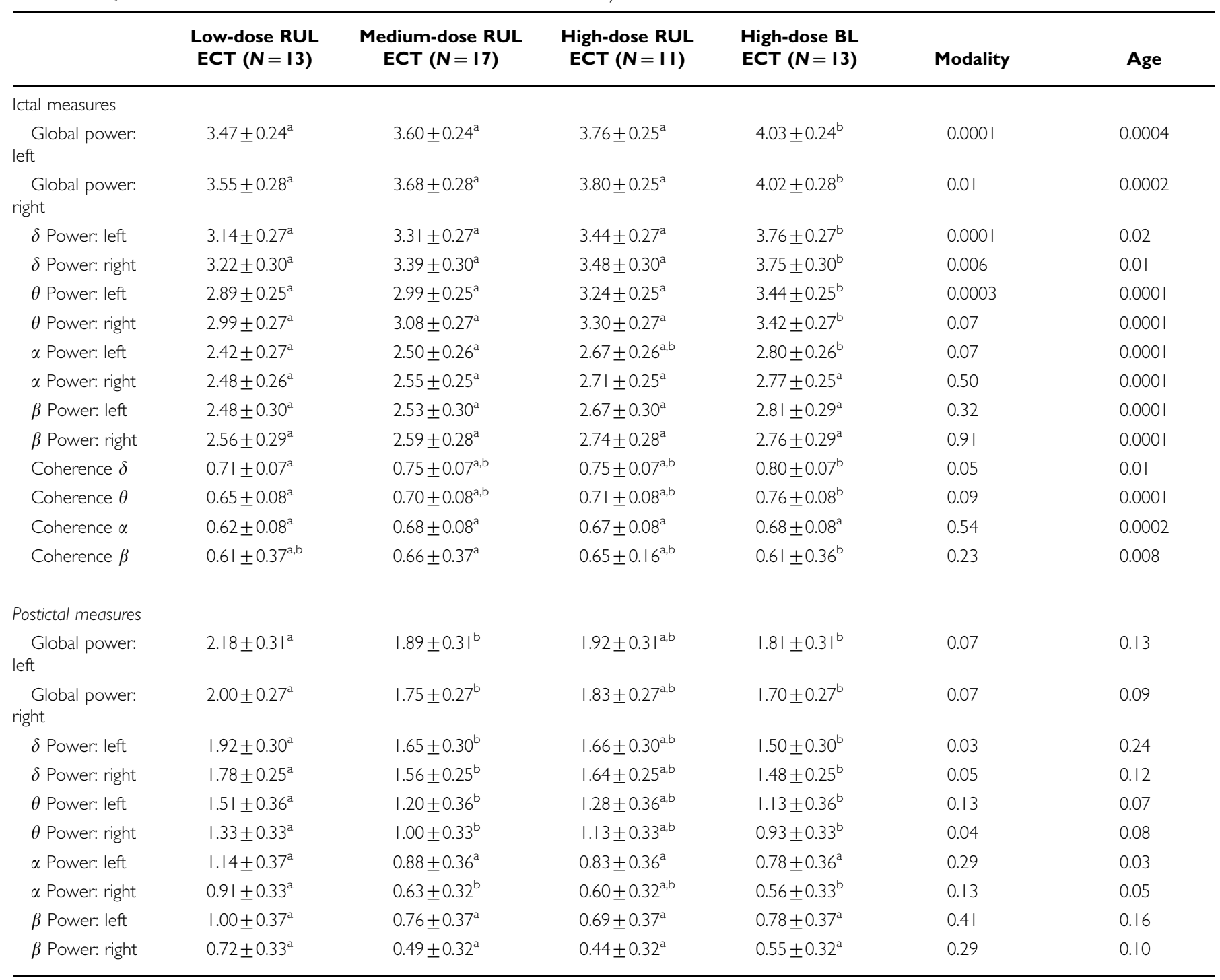

Values for each modality are least-squares adjusted means $\pm S D$, covarying for age. Cells that share the same superscript did not differ significantly in post hoc analyses, while cells with different superscripts differed significantly. 
addition, age had strong associations with ictal power and coherence. Older patients consistently showed lower ictal power and less coherence across all frequency bands.

\section{Relations Between Efficacy and Quantitative EEG Measures}

The percentage change in HRSD scores over the ECT course was added as a second covariate and the ANCOVAs were repeated. The significance levels for the effects of ECT modality, age, and clinical improvement are presented in Table 5. Controlling for modality and age effects, greater clinical improvement was associated with great global ictal power (left and right), greater ictal $\delta$ power (left and right), and greater $\delta$ coherence values. For the postictal quantitative measures, greater EEG suppression (ie less power) was associated with greater clinical improvement for global power (left), $\theta$ power (left and right), and $\alpha$ power (left and right). When these ANCOVAs were repeated, restricting the sample to the 41 RUL ECT patients, there was little change in the pattern of significant effects associated with clinical improvement.

Of the initial responders, seven of $32(21.9 \%)$ no longer met response criteria 1 week following the acute treatment course. Table 5 also presents the results of ANCOVAs in total sample substituting the degree of improvement 1 week following ECT (relative to pre-ECT baseline) for the same measure assessed immediately following ECT. The significant relations observed before were now essentially absent. Thus, the quantitative EEG measures were more strongly related to immediate clinical outcome than the improvement seen 1 week following ECT.

\section{Relations Between ECT Modality, Efficacy, and Observer EEG Ratings}

The ANCOVAs on observer ratings with ECT modality as a between-subject factor and age as a covariate yielded only

Table 5 Significance Levels of Modality, Age, and Degree of Clinical Improvement Immediately and I Week Following ECT on Quantitative EEG Measures

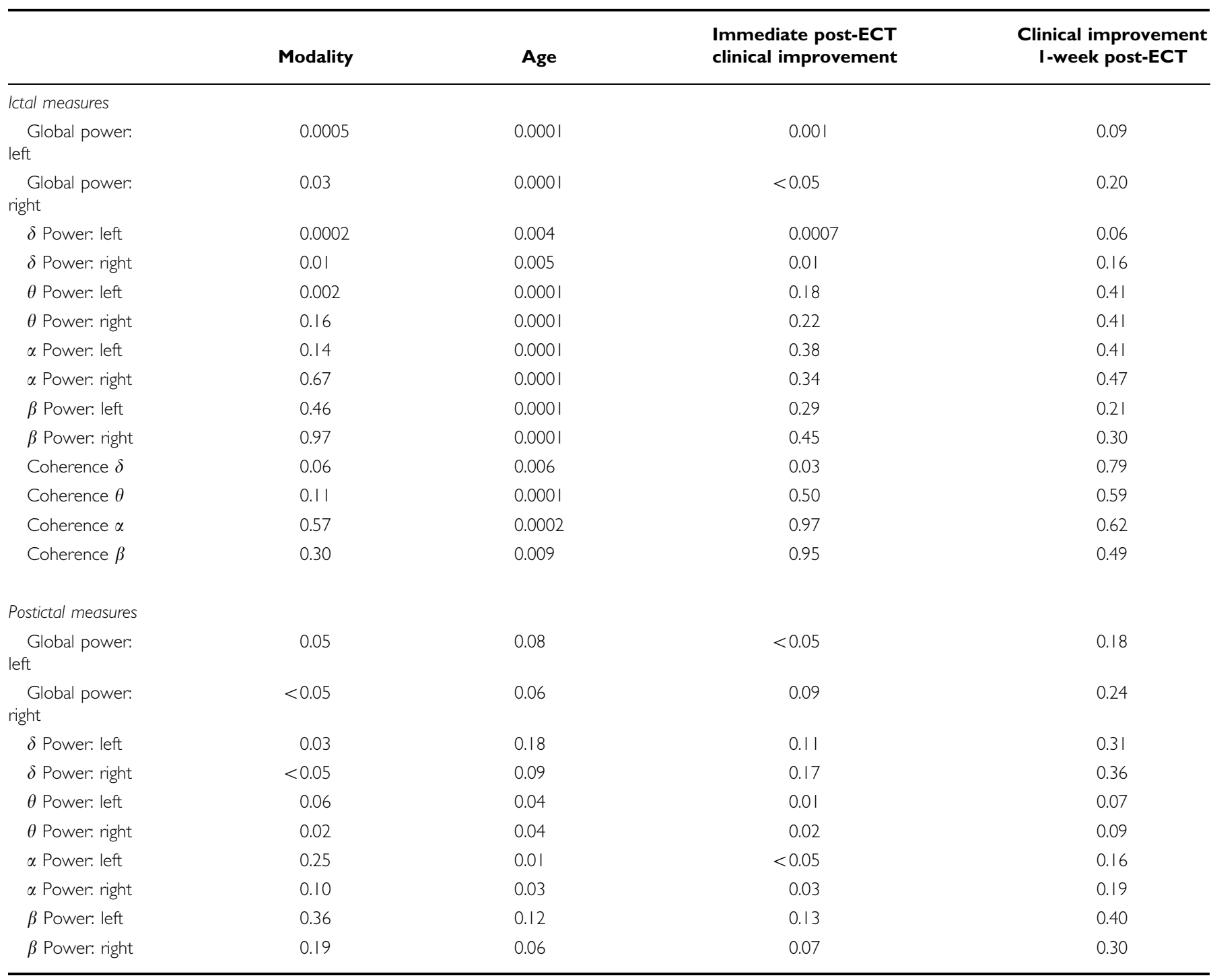


one significant effect of modality. The treatment groups differed in the ratings of the abruptness of seizure termination, $\mathrm{F}(3,49)=2.84, P=0.048$, with the high dosage RUL ECT group having the least abrupt termination and differing from both low dosage RUL ECT and high dosage BL ECT. As with the quantitative measures, the RUL ECT groups could not be distinguished in any of the other observer ratings. However, unlike the quantitative indices, where BL ECT was distinct in several measures, the ECT modalities showed little differentiation in the observer ratings.

The same ANCOVA models used for the quantitative indices tested the relations between the observer ratings and the immediate and 1-week post-ECT measures of symptomatic improvement (Table 6). The observer ratings of ictal strength and ictal pattern were significantly related to improvement at both post-ECT time-points.

The ANCOVAs were repeated restricting the sample to patients treated with RUL ECT. Ictal strength, $\mathrm{F}(1,40)=12.21, \quad P=0.001$, and pattern, $\mathrm{F}(1,40)=8.20$, $P=0.007$, continued to be associated with immediate post-ECT clinical improvement, and amplitude reduction and abruptness of seizure offset showed similar trends ( $P$ 's $<0.10)$. At 1 week following ECT, ratings of ictal strength, $\mathrm{F}(1,40)=5.82, \quad P=0.02$, and pattern, $\mathrm{F}(1,40)=4.69$, $P=0.04$, were still significantly related to extent of symptomatic change.

\section{Prediction of Remission Status}

The three quantitative EEG indices (left-sided ictal $\delta$ power, ictal $\delta$ coherence, and left-sided postictal $\theta$ power) and two observer ratings (ictal strength and pattern) that showed the strongest relations with clinical improvement, as well as age, were used to develop the discriminant functions predicting the remission outcomes. Classification accuracy was $80.7 \%$ in identifying initial remitters and $69.6 \%$ for initial nonremitters, $\chi^{2}(1)=13.79, P=0.0002, \kappa=0.50$. Greater ictal $\delta$ power, $\mathrm{F}(1,52)=10.79, P<0.002$, and less postictal $\theta$ power, $\mathrm{F}(1,52)=4.05, P<0.05$, significantly contributed to prediction. Classification accuracy was $78.3 \%$ in identifying final remitters and $51.6 \%$ for final nonremitters, $\chi^{2}(1)=4.96, P<0.03, \kappa=0.28$. Again, greater ictal $\delta$ power, $\mathrm{F}(1,52)=4.17, \quad P<0.05$, and less postictal $\theta$ power, $\mathrm{F}(1,52)=4.52, P<0.04$, significantly contributed to prediction. The findings were essentially unaltered when restricting the sample to the patients treated with RUL ECT. Overall classification accuracy was $75.6 \%$ for initial remission, $\chi^{2}(1)=10.90, P=0.001, \kappa=0.50$, and dropped to $58.5 \%$ for final remission, $\chi^{2}(1)=2.80, P=0.09, \kappa=0.26$.

\section{Relations Between Cognitive Side Effects and EEG Measures}

There was a significant effect of treatment modality in the ANCOVAs conducted on the four measures of acute cognitive effects and five measures of short-term cognitive effects (all $P$ 's $<0.05$ ). In each instance, BL ECT produced the greatest acute and short-term deficits. Associations with the quantitative EEG indices and observer ratings were sparse and no EEG variable showed a significant association with more than one cognitive measure. Thus, there was no evidence that peri-ictal EEG features were associated with the acute or short-term cognitive effects of ECT.

\section{Ancillary Analyses: Peak EEG Expression and Restriction to Early Treatments}

The ancillary analyses using peak ictal power values duplicated the findings of the primary analyses, without exception. The correlations between peak value and the corresponding measure averaged across the entire seizure ranged from 0.92 to 0.95 . Restricting the data to the second and third treatments did not produce any new significant effects and the strength of associations seen in the primary analyses diminished. There were only two effects of treatment modality in the ANCOVAs on the quantitative measures, involving ictal left global power, $\mathrm{F}(3,49)=3.06$, $P<0.04$, and $\theta$ coherence, $\mathrm{F}(3,49)=4.37, P=0.008$. As in the primary analyses, only abruptness of termination showed an effect of modality in the observer ratings, $\mathrm{F}(3,49)=3.36, P<0.03$. When clinical improvement immediately following ECT was added as a covariate to the ANCOVAs, for the quantitative measures only left and right

Table 6 Significance Levels of Modality, Age, and Degree of Clinical Improvement Immediately and I Week Following ECT on Visual EEG Ratings

\begin{tabular}{|c|c|c|c|c|}
\hline & Modality & Age & $\begin{array}{l}\text { Immediate post-ECT clinical } \\
\text { improvement }\end{array}$ & $\begin{array}{c}\text { Clinical improvement I-week } \\
\text { post-ECT }\end{array}$ \\
\hline Seizure duration & 0.10 & 0.03 & 0.43 & 0.40 \\
\hline Ictal pattern & 0.84 & 0.0001 & 0.01 & 0.05 \\
\hline $\begin{array}{l}\text { Amplitude } \\
\text { reduction }\end{array}$ & 0.54 & 0.57 & 0.09 & 0.38 \\
\hline $\begin{array}{l}\text { Abrupt } \\
\text { termination }\end{array}$ & 0.04 & 0.0007 & 0.50 & 0.45 \\
\hline Fit switch present & 0.45 & 0.45 & 0.76 & 0.64 \\
\hline Postictal silence & 0.42 & 0.19 & 0.52 & 0.54 \\
\hline
\end{tabular}


ictal $\delta$ power were related to outcome $\left(P^{\prime} s \leqslant 0.03\right)$, while among the observer ratings, ictal strength, frequency reduction, and amplitude reduction were associated with degree of improvement $(P$ 's $\leqslant 0.05)$. For the 1 -week postECT clinical assessment, none of the quantitative measures had significant relationships, but the observer ratings of ictal strength and frequency and amplitude reduction continued to show significant associations. In predicting initial remission, only $63.5 \%$ of patients were correctly classified by the discriminant function, $\chi^{2}(1)=3.18$, $P=0.07, \kappa=0.25$, with only ictal $\delta$ power significantly contributing, $\mathrm{F}(1,53)=5.11, P<0.03$. Accuracy dropped to $49.1 \%$ in predicting final remission status, $\chi^{2}(1)=0.17$, $P=0.68, \kappa=0.05$. In both sets of ancillary sets, none of the EEG features showed consistent relations with cognitive side effects. When restricting the data to the second and third treatments the magnitude of mean differences among the modalities and the $\beta$ weights in the regressions on clinical outcome were comparable to those in the primary analyses, but the within-group variances were increased. This indicated that the more powerful effects observed in the primary analyses were due to enhanced reliability of measurement due to repeated observation rather than an increase in the strength of associations over the treatment course.

\section{DISCUSSION}

Several studies have suggested that EEG measures of ECT seizure expression may be useful in assessing treatment adequacy and guiding changes in treatment delivery, specifically stimulus dosing (Krystal et al, 1995, 1996, 1998, 2000a, b; Folkerts, 1996; McCall et al, 1996a, 1998). Quantitative analyses and observer ratings have consistently shown that an ineffective form of treatment, RUL ECT administered just above ST $(1 \times \mathrm{ST})$, has diminished ictal amplitude and reduced postictal suppression compared to RUL ECT at higher dosage relative to ST (Krystal et al, 1993, 1995; Nobler et al, 1993, 2000; McCall et al, 1996b, 1998; Luber et al, 2000).

The conditions were optimized in this study for detecting relationships between EEG features and ECT modality and behavioral outcomes. EEG was collected at all treatments using standardized site preparation, impedance testing, and other quality control procedures. Both quantitative indices and observer ratings were examined. The pattern of strong and specific associations between these two measurement domains supported the reliability and validity of the indices. Patients had a substantial medication washout prior to ECT. The RUL ECT conditions varied markedly in stimulus dosage, with groups treated at $1.5,2.5$, and $6 \times$ ST. The ECT modalities had marked and distinct differences in their effects on efficacy and cognition, with high dosage RUL and BL ECT equivalent in therapeutic effects, but BL ECT producing more severe cognitive deficits.

Nonetheless, the three RUL ECT conditions showed minimal differences in their effects on peri-ictal EEG features. This negative finding indicates that while barely suprathreshold RUL ECT $(1.0 \times \mathrm{ST})$ has blunted EEG expression, the effects of electrical dosage on EEG features begin to saturate when RUL ECT is only modestly above ST (ie $1.5 \times \mathrm{ST}$ ). In other words, the window for electrical dosage effects on EEG seizure expression is considerably narrower than that for efficacy. In the only other study to examine a wide dosing range for RUL ECT, (McCall et al, 1998), using a within-patient randomized design, found that ratings of seizure regularity increased from barely suprathreshold to moderate dosage RUL ECT, but did not distinguish moderate and high dosage RUL ECT. Given this lack of differentiation, it does not seem likely that analysis of EEG seizure characteristics will have much utility in guiding stimulus dosing with RUL ECT. In contrast, compared to each RUL ECT condition, BL ECT had greater ictal power in the slow frequency bands, and greater $\delta$ coherence and postictal suppression.

Research in this area has attempted to identify EEG features sensitive to both the extent to which the electrical dosage of ongoing treatment exceeds ST and the likelihood of remission at the end of the ECT course. The EEG features found in several studies to predict positive clinical outcome have been consistent with those distinguishing barely suprathreshold RUL ECT from higher dosage RUL or BL ECT. Thus, at least when using RUL ECT, it was assumed that manifestation of EEG characteristics predictive of poor outcome should trigger consideration of a dosage increment, thereby improving EEG seizure expression and, more critically, enhancing efficacy. However, prior research on dosage effects was limited by a focus on the differences of RUL ECT at barely suprathreshold dosage compared to higher dosage conditions (Krystal et al, 1993, 1995, 2000b; Nobler et al, 1993; Luber et al, 2000). Especially problematic for this account have been reports that the same peri-ictal features are also associated with the efficacy of BL ECT (Nobler et al, 1993, 2000; Folkerts, 1996; Hrdlicka et al, 1996; Suppes et al, 1996), which is far less sensitive than RUL ECT to effects of dosage on efficacy (Sackeim et al, 1987a, 1993).

We found that peri-ictal EEG features did not distinguish forms of RUL ECT that differed markedly both in electrical dosage and efficacy. Nonetheless, there were significant associations between EEG features and clinical outcome, independent of ECT modality. We suggest that the field has misinterpreted these associations as reflecting the therapeutic potency of ongoing treatment. Instead, we suggest that these associations derive from differences among patients in neurophysiological response to seizure provocation and that these individual differences, independent of ECT treatment parameters, are linked to therapeutic outcome.

ECT seizure induction can be viewed as a challenge test, like that used to assess glucose intolerance or early escape from dexamethasone suppression. While factors such as dose of the challenge agent may impact on outcomes, challenge procedures are most commonly used to identify individual differences in biological vulnerabilities or disease states (eg glucose intolerance or diabetes, HPA axis abnormality). The method of limits, titration procedure used to quantify ST illustrates how seizure induction serves as a challenge procedure. Under general anesthesia, stimulus dosage is systematically increased until a generalized seizure is induced, thereby quantifying ST (Sackeim et al, 1987b). The obtained threshold is influenced by technical parameters of the challenge and individual 
differences factors. ST is markedly higher with BL than RUL ECT (Sackeim et al, 1987a, b; McCall et al, 1993), with sine wave than brief pulse stimulation (Weiner, 1980), and with brief pulse than ultra-brief pulse stimulation. There are also marked individual differences in ST, which increases with age and is higher in men than women (Sackeim et al, 1987b; Coffey et al, 1995; Colenda and McCall, 1996; Lisanby et al, 1996; Boylan et al, 2000). ST also has an inverse association with ECT seizure duration, validating that this measure reflects individual differences in neural excitability. Of note, manipulations that alter the minimum threshold for a seizure are often distinct from those that alter seizure duration (Green et al, 1982a; Sackeim et al, 1991). The fact that ST predicts seizure duration suggests that its physiological bases comprise more than one dimension of neural excitability.

There are also marked individual differences in ECT seizure expression. In the current study and previous work, patient age strongly predicted variation in peri-ictal measures, demonstrating that a considerable amount of the variability is related to the person and not simply ECT technique. In an earlier sample using a 19-lead EEG montage, in addition to age, we reported that baseline severity of depressive symptoms and initial ST predicted quantitative EEG measures of ECT seizure expression (Nobler et al, 2000). In the present study, after controlling for ECT modality, age, and degree of clinical improvement immediately following ECT, baseline HRSD scores were significantly associated with all 10 quantitative measures of ictal power and with the observer ratings of ictal strength and pattern, the abruptness of seizure termination, and postictal silence (data not shown). Patients with greater depression severity had higher amplitude seizure expression, more abrupt termination, and ratings of greater postictal suppression. Initial ST had a more limited pattern of association. Higher ST was associated with less ictal leftsided global and $\delta$ power. The associations with age, depression severity, and initial ST must reflect intrinsic individual differences, as opposed to treatment parameters, since patients were randomized to the treatment conditions. Similarly, it is likely that patients who remit following ECT differ in seizure manifestations from nonremitters because their differences in neurophysiological response to seizure induction reflect their potential for antidepressant effects, and not necessarily because the treatments they are receiving differ in potency.

The EEG features associated with positive clinical outcome were consistent across the analyses averaging over the treatment course and those restricted to the second and third treatments. Maintenance of these associations at the early treatments ruled out the possibility that the relationships reflected variability in seizure expression modulated by clinical state change. The associations were more robust when examining clinical outcome immediately following ECT compared to 1 week later. The weakening of associations at the 1-week assessment was due to some patients showing symptomatic worsening during the week after ECT. This phenomenon is commonly observed and approximately $15 \%$ of patients who initially meet remission criteria no longer do so 1 week after ECT (Sackeim et al, 1987a, 1993, 2000b). That individual differences in seizure expression predicted immediate but not delayed clinical outcome suggests that differences in the physiological expression of seizures reflect on the potential for a pronounced antidepressant effect but not its durability.

Superior outcome was associated with greater ictal power in the $\delta$ band, greater ictal coherence in $\delta$, and greater postictal suppression. We suggest that these features reflect the strength of the endogenous inhibitory processes that terminate seizures (Sackeim et al, 1983; Sackeim, 1999). The spike and slow wave complexes during generalized seizures reflect the intensity of synchronous and opponent excitatory and inhibitory processes. The $\delta$ wave component originates from vertically arranged dipoles in cortical layers II-III and V (Calvet et al, 1964; Petsche et al, 1984; Steriade, 1994), and results from the firing of GABAergic interneurons inhibiting pyramidal cells (Ball et al, 1977; Steriade et al, 1990) and the summation of long-lasting after hyperpolarizations, due to potassium currents in deep cortical pyramidal cells (Schwindt et al, 1989; Neckelmann et al, 2000). In turn, the regional topography of the ictal slow wave activity during ECT-induced seizures (Nobler et al, 2000) closely matches the topography of reduced blood flow and metabolism and increased EEG slow frequency activity during and following the treatment course (Nobler et al, 1994, 2001; Sackeim et al, 1996), indicating persistence of the inhibitory effect. Thus, we offer the hypothesis that individuals who marshal more intense inhibitory processes during and immediately following seizures have greater potential to benefit from ECT, and that these individual differences underlie the associations between peri-ictal EEG measures and ECT clinical outcome. While GABAergic transmission is the leading candidate mediating this effect (Green et al, 1982b; Green, 1986; Sanacora et al, 2003), other inhibitory neurotransmitters and peptides should also be considered.

\section{ACKNOWLEDGEMENTS}

This work was supported in part by R01 MH35636, R01 MH57009, and R01 MH61609 from the National Institute of Mental Health. We thank Linda Fitzsimons, RN, Benjamin Ginsberg, BA, and David Leitman, BA for assistance with data collection.

\section{REFERENCES}

American Psychiatric Association (2001). The Practice of ECT: Recommendations for Treatment, Training and Privileging, 2nd edn American Psychiatric Press: Washington, DC.

Ball CJ, Gloor P, Schaul N (1977). The cortical electromicrophysiology of pathological delta waves in the electroencephalogram of the cat. Electroencephalogr Clin Neuro Physiol 43: 346-361.

Boylan LS, Haskett RF, Mulsant BH, Greenberg RM, Prudic J, Spicknall K et al (2000). Determinants of seizure threshold in ECT: benzodiazepine use, anesthetic dosage, and other factors. J ECT 16: 3-18.

Buschke H (1973). Selective reminding for analysis of memory and learning. J Verbal Learn Verbal Behav 12: 543-550.

Calvet J, Calvet MC, Scherer J (1964). Etude stratigraphique corticale de l'activité EEG spontanée. Electroencephalogr Clin Neurophysiol 17: 109-125.

Coffey CE, Lucke J, Weiner RD, Krystal AD, Aque M (1995). Seizure threshold in electroconvulsive therapy: I. Initial seizure threshold. Biol Psychiatry 37: 713-720. 
Colenda CC, McCall WV (1996). A statistical model predicting the seizure threshold for right unilateral ECT in 106 patients. Convulsive Ther 12: 3-12.

Devanand DP, Bowers MBJ, Hoffman FJJ, Sackeim HA (1989). Acute and subacute effects of ECT on plasma HVA, MHPG, and prolactin. Biol Psychiatry 26: 408-412.

Devanand DP, Lisanby S, Lo ES, Fitzsimons L, Cooper TB, Halbreich U et al (1998). Effects of electroconvulsive therapy on plasma vasopressin and oxytocin. Biol Psychiatry 44: 610-616.

Devanand DP, Shapira B, Petty F, Kramer G, Fitzsimons L, Lerer B et al (1995). Effects of electroconvulsive therapy on plasma GABA. Convulsive Ther 11: 3-13.

Endicott J, Spitzer RL (1978). A diagnostic interview: the schedule for affective disorders and schizophrenia. Arch Gen Psychiatry 35: $837-844$.

Fink M (1979). Convulsive Therapy: Theory and Practice. Raven Press: New York.

Fink M, Nemeroff CB (1989). A neuroendocrine view of ECT. Convulsive Ther 5: 296-304.

Folkerts H (1996). The ictal electroencephalogram as a marker for the efficacy of electroconvulsive therapy. Eur Arch Psychiatry Clin Neurosci 246: 155-164.

Green A (1986). Changes in gamma-aminobutyric acid biochemistry and seizure threshold. Ann NY Acad Sci 462: 105-119.

Green A, Nutt D, Cowen P (1982a). Increased seizure threshold following convulsion. In: Sandler M (ed). Psychopharmacology of Anticonvulsants. Oxford University Press: Oxford. pp 16-26.

Green A, Sant K, Bowdler J, Cowen P (1982b). Further evidence for a relationship between changes in GABA concentration in rat brain and enhanced monoamine-mediated behavioural responses following repeated electroconvulsive shock. Neuropharmacology 21: 981-984.

Hamilton M (1967). Development of a rating scale for primary depressive illness. Br J Soc Clin Psychol 6: 278-296.

Heikman P, Kalska H, Katila H, Sarna S, Tuunainen A, Kuoppasalmi K (2002). Right unilateral and bifrontal electroconvulsive therapy in the treatment of depression: a preliminary study. J ECT 18: 26-30.

Hrdlicka M, Moran M, Vachutka J, Blatny M, Rozinkova J (1996). EEG in electroconvulsive therapy: is more intensive paroxysmal activity associated with a higher therapeutic response? Neuropsychobiology 33: 138-141.

Huberty CJ (1984). Issues in the use and interpretation of discriminant analysis. Psychol Bull 95: 156-171.

Kronfol Z, Hamdan-Allen G, Goel K, Hill EM (1991). Effects of single and repeated electroconvulsive therapy sessions on plasma ACTH, prolactin, growth hormone and cortisol concentrations. Psychoneuroendocrinology 16: 345-352.

Krystal AD, Coffey CE, Weiner RD, Holsinger T (1998). Changes in seizure threshold over the course of electroconvulsive therapy affect therapeutic response and are detected by ictal EEG ratings. J Neuropsychiatry Clin Neurosci 10: 178-186.

Krystal AD, Holsinger T, Weiner RD, Coffey CE (2000a). Prediction of the utility of a switch from unilateral to bilateral ECT in the elderly using treatment 2 ictal EEG indices. J ECT 16: 327-337.

Krystal AD, Weiner RD (1994). ECT seizure therapeutic adequacy. Convulsive Ther 10: 153-164.

Krystal AD, Weiner RD, Coffey CE (1995). The ictal EEG as a marker of adequate stimulus intensity with unilateral ECT. J Neuropsychiatry Clin Neurosci 7: 295-303.

Krystal AD, Weiner RD, Coffey CE, McCall WV (1996). Effect of ECT treatment number on the ictal EEG. Psychiatry Res 62: 179-189.

Krystal AD, Weiner RD, Lindahl V, Massie R (2000b). The development and retrospective testing of an electroencephalographic seizure quality-based stimulus dosing paradigm with ECT. J ECT 16: 338-349.
Krystal AD, Weiner RD, McCall WV, Shelp FE, Arias R, Smith P (1993). The effect of ECT stimulus dose and electrode placement on the ictal electroencephalogram: an intraindividual crossover study. Biol Psychiatry 34: 759-767.

Lisanby SH, Devanand DP, Nobler MS, Prudic J, Mullen L, Sackeim HA (1996). Exceptionally high seizure threshold: ECT device limitations. Convulsive Ther 12: 156-164.

Lisanby SH, Devanand DP, Prudic J, Pierson D, Nobler MS, Fitzsimons L et al (1998). Prolactin response to electroconvulsive therapy: effects of electrode placement and stimulus dosage. Biol Psychiatry 43: 146-155.

Luber B, Nobler MS, Moeller JR, Katzman GP, Prudic J, Devanand DP et al (2000). Quantitative EEG during seizures induced by electroconvulsive therapy: relations to treatment modality and clinical features. II. Topographic analyses. J ECT 16: 229-243.

McCall WV, Colenda CC, Farah BA (1996a). Ictal EEG regularity declines during a course of RUL ECT. Convulsive Ther 12: 213-216.

McCall WV, Farah A, Reboussin DM (1995). Can we teach psychiatric residents to rate seizure regularity? Convulsive Ther 11: 248-252.

McCall WV, Reboussin DM, Weiner RD, Sackeim HA (2000). Titrated moderately suprathreshold $v s$ fixed high-dose right unilateral electroconvulsive therapy: acute antidepressant and cognitive effects. Arch Gen Psychiatry 57: 438-444.

McCall WV, Robinette GD, Hardesty D (1996b). Relationship of seizure morphology to the convulsive threshold. Convulsive Ther 12: 147-151.

McCall WV, Shelp FE, Weiner RD, Austin S, Norris J (1993). Convulsive threshold differences in right unilateral and bilateral ECT. Biol Psychiatry 34: 606-611.

McCall WV, Sparks W, Jane J, Rosenquist PB, Colenda CC, Reboussin DM (1998). Variation of ictal electroencephalographic regularity with low-, moderate-, and high-dose stimuli during right unilateral electroconvulsive therapy. Biol Psychiatry 43: 608-611.

McElhiney MC, Moody BJ, Steif BL, Prudic J, Devanand DP, Nobler MS et al (1995). Autobiographical memory and mood: effects of electroconvulsive therapy. Neuropsychology 9: 501-517.

Neckelmann D, Amzica F, Steriade M (2000). Changes in neuronal conductance during different components of cortically generated spike-wave seizures. Neuroscience 96: 475-485.

Nobler MS, Luber B, Moeller JR, Katzman GP, Prudic J, Devanand DP et al (2000). Quantitative EEG during seizures induced by electroconvulsive therapy: relations to treatment modality and clinical features. I. Global analyses. J ECT 16: 211-228.

Nobler MS, Oquendo MA, Kegeles LS, Malone KM, Campbell CC, Sackeim HA et al (2001). Decreased regional brain metabolism after ECT. Am J Psychiatry 158: 305-308.

Nobler MS, Sackeim HA, Prohovnik I, Moeller JR, Mukherjee S, Schnur DB et al (1994). Regional cerebral blood flow in mood disorders, III. Treatment and clinical response. Arch Gen Psychiatry 51: 884-897.

Nobler MS, Sackeim HA, Solomou M, Luber B, Devanand DP, Prudic J (1993). EEG manifestations during ECT: effects of electrode placement and stimulus intensity. Biol Psychiatry 34: 321-330.

Ohman R, Walinder J, Balldin J, Wallin L (1976). Prolactin response to electroconvulsive therapy. Lancet 2: 936-937.

Ottosson J-O (1960). Experimental studies of the mode of action of electroconvulsive therapy. Acta Psychiatr Scand 145, (Suppl) $1-141$.

Petsche H, Pockberger H, Rappelsberger P (1984). On the search for the sources of the electroencephalogram. Neuroscience 11: $1-27$.

Prudic J, Sackeim HA, Devanand DP, Krueger RB, Settembrino JM (1994). Acute cognitive effects of subconvulsive electrical stimulation. Convulsive Ther 10: 4-24. 
Rey A (1941). L'examen psychologique dans les cas d'encephalopathie traumatique. Arch Psychol 28: 286-340.

Robin A, De Tissera S (1982). A double-blind controlled comparison of the therapeutic effects of low and high energy electroconvulsive therapies. Br J Psychiatry 141: 357-366.

Sackeim HA (1986). Acute cognitive side effects of ECT. Psychopharmacol Bull 22: 482-484.

Sackeim HA (1999). The anticonvulsant hypothesis of the mechanisms of action of ECT: current status. J ECT 15: 5-26.

Sackeim HA (2001). The definition and meaning of treatmentresistant depression. J Clin Psychiatry 62: 10-7-10-17.

Sackeim HA, Decina P, Kanzler M, Kerr B, Malitz S (1987a). Effects of electrode placement on the efficacy of titrated, low-dose ECT. Am J Psychiatry 144: 1449-1455.

Sackeim HA, Decina P, Prohovnik I, Malitz S (1987b). Seizure threshold in electroconvulsive therapy. Effects of sex, age, electrode placement, and number of treatments. Arch Gen Psychiatry 44: 355-360.

Sackeim HA, Decina P, Prohovnik I, Malitz S, Resor SR (1983). Anticonvulsant and antidepressant properties of electroconvulsive therapy: a proposed mechanism of action. Biol Psychiatry 18: $1301-1310$.

Sackeim HA, Devanand DP, Nobler MS (1995). Electroconvulsive therapy. In: Bloom F, Kupfer D (eds). Psychopharmacology: The Fourth Generation of Progress. Raven: New York. pp 1123-1142.

Sackeim HA, Devanand DP, Prudic J (1991). Stimulus intensity, seizure threshold, and seizure duration: impact on the efficacy and safety of electroconvulsive therapy. Psychiatr Clin North Am 14: 803-843.

Sackeim HA, Luber B, Katzman GP, Moeller JR, Prudic J, Devanand DP et al (1996). The effects of electroconvulsive therapy on quantitative electroencephalograms. Relationship to clinical outcome. Arch Gen Psychiatry 53: 814-824.

Sackeim HA, Luber B, Moeller JR, Prudic J, Devanand DP, Nobler MS (2000a). Electrophysiological correlates of the adverse cognitive effects of electroconvulsive therapy. J ECT 16: 110-120.

Sackeim HA, Portnoy S, Neeley P, Steif BL, Decina P, Malitz S (1986). Cognitive consequences of low-dosage electroconvulsive therapy. Ann NY Acad Sci 462: 326-340.

Sackeim HA, Prudic J, Devanand DP, Kiersky JE, Fitzsimons L, Moody BJ et al (1993). Effects of stimulus intensity and electrode placement on the efficacy and cognitive effects of electroconvulsive therapy. $N$ Engl J Med 328: 839-846.

Sackeim HA, Prudic J, Devanand DP, Nobler MS, Lisanby SH, Peyser S et al (2000b). A prospective, randomized, double-blind comparison of bilateral and right unilateral electroconvulsive therapy at different stimulus intensities. Arch Gen Psychiatry 57: 425-434.

Sanacora G, Mason GF, Rothman DL, Hyder F, Ciarcia JJ, Ostroff $\mathrm{RB}$ et al (2003). Increased cortical GABA concentrations in depressed patients receiving ECT. Am J Psychiatry 160: 577-579.
Schwindt PC, Spain WJ, Crill WE (1989). Long-lasting reduction of excitability by a sodium-dependent potassium current in cat neocortical neurons. J Neurophysiol 61: 233-244.

Scott AI (1989). Which depressed patients will respond to electroconvulsive therapy? The search for biological predictors of recovery. Br J Psychiatry 154: 8-17.

Sobin C, Sackeim HA, Prudic J, Devanand DP, Moody BJ, McElhiney MC (1995). Predictors of retrograde amnesia following ECT. Am J Psychiatry 152: 995-1001.

Spitzer RL, Endicott J, Robins E (1978). Research diagnostic criteria: rationale and reliability. Arch Gen Psychiatry 35: 773-782.

Spreen O, Strauss E (1998). A Compendium of Neuropsychological Tests: Administration, Norms, and Commentary. Oxford University Press: New York.

Staton RD, Hass PJ, Brumback RA (1981). Electroencephalographic recording during bitemporal and unilateral nondominant hemisphere (Lancaster position) electroconvulsive therapy. J Clin Psychiatry 42: 264-269.

Steriade M (1994). Cellular substrates of brain rhythms. In: Lopes da Silva FH (ed). Electroencephalography: Basic Principles, Clinical Applications, and Related Fields, 3rd edn Williams \& Wilkins: Baltimore, MD. pp 27-62.

Steriade M, Gloor P, Llinás RR, Lopes da Silva FH, Mesulam M-M (1990). Basic mechanisms of cerebral rhythmic activities. Electroencephalogr Clin Neurophysiol 76: 481-508.

Stern Y, Sano M, Pauson J, Mayeux R (1987). Modified mini-mental status examination: validity and reliability. Neurology 37: 179.

Sundblom DM, Heikman P, Naukkarinen H, Fyhrquist F (1999). Blood concentrations of vasopressin, neuropeptide $\mathrm{FF}$ and prolactin are increased by high-dose right unilateral ECT. Peptides 20: 319-326.

Suppes T, Webb A, Carmody T, Gordon E, Gutierrez-Esteinou R, Hudson JI et al (1996). Is postictal electrical silence a predictor of response to electroconvulsive therapy? J Affect Disord 41: 55-58.

Tew Jr JD, Mulsant BH, Haskett RF, Dolata D, Hixson L, Mann JJ (2002). A randomized comparison of high-charge right unilateral electroconvulsive therapy and bilateral electroconvulsive therapy in older depressed patients who failed to respond to 5 to 8 moderate-charge right unilateral treatments. J Clin Psychiatry 63: $1102-1105$.

Weiner RD (1980). ECT and seizure threshold: effects of stimulus wave form and electrode placement. Biol Psychiatry 15: 225-241.

Weiner RD (1982). Electroencephalographic correlates of ECT. Psychopharmacol Bull 18: 78-81.

Whalley L, Eagles J, Bowler G, Bennie J, Dick H, McGuire R et al (1987). Selective effects of ECT on hypothalamic-pituitary activity. Psychol Med 17: 319-328.

Zis AP, Yatham LN, Lam RW, Clark CM, Srisurapanont M, McGarvey K (1996). Effect of stimulus intensity on prolactin and cortisol release induced by unilateral electroconvulsive therapy. Neuropsychopharmacology 15: 263-270. 